\title{
Decision analysis for manufacturers to carry out emission reduction through technology Innovation under carbon emission constraints
}

\author{
Mao chaoyan \\ (Chongqing Jiaotong University, Chongqing 400074,China)
}

Key words: Carbon emissions, Emission reduction,Technology innovation,Supply chain anagement

\begin{abstract}
In view of a secondary supply chain system composed of one manufacturer and one retailer, the paper builds the judgment model about how the prevailing manufacturer can carry out emission reduction through technology innovation under carbon emission constraints by taking Stackelberg Game theory, and compares the impacts of the manufacturer decisions on the prices and profits of the manufacturer and the retailer. The results show that the prices and profits of the manufacturer and the retailer are the higher with technology innovation than without technology innovation under certain conditions, and are affected by the consumer preference for low carbon, market size and investment size parameter. In addition, there is a threshold value about the optimal carbon emissions caps and the policy can encourage technology innovation and carbon reduction within the threshold value. At last the results are verified by the sample application.
\end{abstract}

\section{Introduction}

Domestic and foreign scholars have preliminary study on the carbon constraints manufacturer's production decisions. Yalabik etc ${ }^{[1]}$ explores the emissions regulations, consumer demand and the influence of enterprise competition to manufacturers emission reduction technology investment .Hua etc ${ }^{[2]}$ conducted a comparative study of the carbon emissions of EOQ model and traditional EOQ model constraints,given the manufacturer's optimal order quantity under constraints of carbon emission, analyzed the influence of carbon trading, carbon pricing, carbon quotas on the manufacturers ordering quantity, carbon emissions and total cost.However,the literature does not consider the background of supply chain. In practice the manufacturer's emission reduction technology innovation will inevitably an important impact on the decision-making of the retailer.

Du etc ${ }^{[3]}$ analyzed in two-stage supply chain that be composed by a single carbon-dependent manufacturers and a single carbon emissions permits suppliers, the impact of cap-and-trade mechanism to manufacturer decision and supply chain performance,also gived the optimal production quantity and the supplier manufacturer's optimal license price. Ghosh etc ${ }^{[4]}$ in the global apparel supply chain background, analyzed and compared the cooperation of manufacturers and retailers under different channel power structures on the influence of emission reduction technology innovation, pricing and profit. However,the literature does not discuss the manufacturer's carbon emissions. Domestic scholars from the perspective of supply chain, more is to consider the carbon emissions policy on supply chain business decisions, but do not to consider the issue of emission reduction technology innovation.

The paper assuming the existence of mandatory emission reduction policy constraints, introducting emission reduction of technological innovation level parameters in the demand function, using stackelberg game theory to build the judgment model about the dominant manufacturer whether the implementation of emission reduction technology innovation profitable decision, aslo analysising the impact of mandatory emission reduction policies on enterprise innovation and carbon emissions.

\section{Problem description and hypothesis}

Suppose in the market, there is a simple supply chain composed of one manufacturer and one retailer, manufacturer is the leader of the Stackelberg game, the retailer is the follower. If manufacturers only produce one product, the unit production cost is $c_{m}$, providing products to 
retailers at wholesale prices is $w$,carbon emissions per unit production is $E$, the level of emission reduction technology innovation is $\theta, \theta \in[0,1]$. Retailers sell products at retail price $p$, the cost of sales is $c_{r}$, The assumption that the consumers have low-carbon product preferences, the form of the demand function can be expressed as $D(p, \theta)=\alpha-\beta p+\mu \theta, \alpha, \beta, \mu$ is a constant and $\alpha, \beta, \mu>0$, among them $\alpha$ is the market capacity; $\beta$ represents the consumer price sensitivity, $\mu$ represents consumers' sensitivity to the level of emission reduction technology innovation.

In general, the higher level of the manufacturer's emission reduction technological innovation $\theta$, the carbon emissions of per unit product $(E)$ is more lower, assumed that there is $E=h-l \theta, h, l$ is constant and $h, l>0$, wherein, $h$ represents the initial emissions manufacturer, $l$ represents sensitive coefficient of emissions to the level of innovation,assumes that the manufacturer reduction technology investment as a function of $C=I \theta^{2}$, here $I$ represents the scale coefficient and $I>0$. Assumed that there is mandatory emissions reduction policy constraints, $E_{\text {cap }}$ represents total carbon emission's upper limits of the policy giving to manufacturers. ${ }^{N}$ represents that the manufacturer does not implement emission reduction technology innovation, $G$ represents that the manufacturer intends to implement emission reduction technology innovation.

\section{Supply chain decisions of manufacturers have no innovation}

When manufacturers have no innovation, i.e. $\theta=0$, the demand function can be expressed as $D\left(p^{N}\right)=\alpha-\beta p^{N}$.In decentralized decision-making, the game is Stackelberg game between dominant manufacturers and retailers,the method is reverse solution. Assumed that manufacturer's wholesale price is $w$, retailers according to their expected profit maximization principle to determine the optimal retail price is $p$; and then the manufacturer according to the retailer's reaction to develop optimal wholesale price is $w$.

Retailer's profit function is: $\pi_{r}^{N}=D\left(p^{N}\right)\left(p^{N}-w^{N}-c_{r}\right)=\left(\alpha-\beta p^{N}\right)\left(p^{N}-w^{N}-c_{r}\right)$

Manufacturer's profit function is: $\pi_{m}^{N}=D\left(p^{N}\right)\left(w^{N}-c_{m}\right)=\left(\alpha-\beta p^{N}\right)\left(w^{N}-c_{m}\right)$

According to the (1)retailers profit maximization of the first-order conditions, can be obtained the retailer's optimal retail price for: ${ }^{N^{*}}=\frac{\alpha+\beta w^{N}+\beta c_{r}}{2 \beta}$

Manufacturers observe the retailer's action according to the (3),according to the retailer's response to develop the optimal wholesale price and optimal carbon emissions per unit product.(3) into (2) can be obtained: $\pi_{m}^{N}=\frac{\left(\alpha-\beta w^{N}-\beta c_{r}\right)\left(w^{N}-c_{m}\right)}{2}$

According to (4) manufacturers' profit maximization of the first-order conditions can calculate the optimal wholesale price of $w^{N^{*}}, w^{N^{*}}$ into (3) can be obtained:

$$
w^{N^{*}}=\frac{\alpha-\beta c_{m}-\beta c_{r}}{2 \beta}+c_{m} ; \quad p^{N^{*}}=\frac{3\left(\alpha-\beta c_{m}-\beta c_{r}\right)}{4 \beta}+\left(c_{m}+c_{r}\right)
$$

Equation (5) into $D\left(p^{N}\right)=\alpha-\beta p^{N}$ demand function can be obtained: $D\left(p^{N}\right)=\frac{\alpha-\beta c_{m}-\beta c_{r}}{4}$

Equation (5) into (1) (2) can be obtained:

$$
\pi_{m}^{N}=\frac{\left(\alpha-\beta c_{m}-\beta c_{r}\right)^{2}}{8 \beta} ; \quad \pi_{r}^{N}=\frac{\left(\alpha-\beta c_{m}-\beta c_{r}\right)^{2}}{16 \beta} ; \pi^{N}=\pi_{m}^{N}+\pi_{r}^{N}=\frac{3\left(\alpha-\beta c_{m}-\beta c_{r}\right)^{2}}{16 \beta}
$$

\section{Supply chain decisions of manufacturers have innovation}

When manufacturers have innovation, the demand function can be expressed as. $D\left(p^{G}, \theta^{G}\right)=\alpha-\beta p^{G}+\mu \theta^{G}$, In decentralized decision-making, the game also is Stackelberg game 
between dominant manufacturers and Assumed that the technical innovation level is $\theta$.

Retailer's profit function is: $\pi_{r}^{G}=D\left(p^{G}, \theta^{G}\right)\left(p^{G}-w^{G}-c_{r}\right)=\left(\alpha-\beta p^{G}+\mu \theta^{G}\right)\left(p^{G}-w^{G}-c_{r}\right)$

Manufacturer's profit function is: $\pi_{m}^{G}=D\left(p^{G}, \theta^{G}\right)\left(w^{G}-c_{m}\right)-I\left(\theta^{G}\right)^{2}=\left(\alpha-\beta p^{G}+\mu \theta^{G}\right)\left(w^{G}-c_{m}\right)-I\left(\theta^{G}\right)^{2}$

According to (8) retailers profit maximization of the first-order conditions, can be obtained the

retailer's optimal retail price for:

$$
p^{G^{*}}=\frac{\alpha+\beta w^{G}+\beta c_{r}+\mu \theta^{G}}{2 \beta}
$$

Manufacturers observe the retailer's action according to (10), according to the retailer's response to develop the optimal wholesale price and optimal level of technical innovation.(10) into (9) can be obtained:

$$
\pi_{m}{ }^{G}=\frac{\left(\alpha-\beta w^{G}-\beta c_{r}+\mu \theta^{G}\right)\left(w^{G}-c_{m}\right)}{2}-I\left(\theta^{G}\right)^{2}
$$

According to (11) manufacturers' profit maximization of the first order conditions,can be obtained: $\quad w^{G^{*}}=\frac{4 I\left(\alpha-\beta c_{m}-\beta c_{r}\right)}{8 \beta I-\mu^{2}}+c_{m} \quad \theta^{G^{*}}=\frac{\mu\left(\alpha-\beta c_{m}-\beta c_{r}\right)}{8 \beta I-\mu^{2}}$

Equation (12) into (10), According to the demand function, can be obtained:

$$
p^{G^{*}}=\frac{6 I\left(\alpha-\beta c_{m}-\beta c_{r}\right)}{8 \beta I-\mu^{2}}+\left(c_{m}+c_{r}\right) ; \quad D\left(p^{G}, \theta^{G}\right)=\frac{2 \beta I\left(\alpha-\beta c_{m}-\beta c_{r}\right)}{8 \beta I-\mu^{2}}
$$

Equation (13) into (8) and (9),can be obtained:

$$
\pi_{m}{ }^{G}=\frac{I\left(\alpha-\beta c_{m}-\beta c_{r}\right)^{2}}{8 \beta I-\mu^{2}} ; \quad \pi_{r}^{G}=\frac{4 \beta I^{2}\left(\alpha-\beta c_{m}-\beta c_{r}\right)^{2}}{\left(8 \beta I-\mu^{2}\right)^{2}} ; \quad \pi^{G}=\pi_{m}{ }^{G}+\pi_{r}^{G}=\frac{I\left(12 \beta I-\mu^{2}\right)\left(\alpha-\beta c_{m}-\beta c_{r}\right)^{2}}{\left(8 \beta I-\mu^{2}\right)^{2}}
$$

\section{Comparative analysis}

By compared with manufacturers without innovation and innovation two circumstances, can be obtained the following proposition:

\section{A. Price comparison}

Proposition 1: when $8 \beta I>\mu^{2}$, there are $w^{G^{*}}>w^{N^{*}}, \quad p^{G^{*}}>p^{N^{*}}$ 。

Proof: According to (5)、（12）、（13）, there are:

$$
w^{G^{*}}-w^{N^{*}}=\frac{\mu^{2}\left(\alpha-\beta c_{m}-\beta c_{r}\right)}{2 \beta\left(8 \beta I-\mu^{2}\right)}>0 \quad p^{G^{*}}-p^{N^{*}}=\frac{3 \mu^{2}\left(\alpha-\beta c_{m}-\beta c_{r}\right)}{4 \beta\left(8 \beta I-\mu^{2}\right)}>0 \text {. Prove that. }
$$

Proposition 1 shows that, when manufacturers implement emission reduction technology innovation, the wholesale prices and retail prices will be higher than the manufacturers do not implement emission reduction technology innovation. This phenomenon is observed in practice, i.e. environmental protection product prices generally will be higher than similar non environmental protection products.

Proposition 2 when $8 \beta I>\mu^{2}, w^{G^{*}}$ and $p^{G^{*}}$ increases with the increasing of $\mu$ or $\alpha$, and decrease with the increasing of $I$.

Proof: According to (12) 、 (13) , there is:

$$
\frac{\partial w^{G^{*}}}{\partial \mu}>0, \frac{\partial w^{G^{*}}}{\partial \alpha}>0, \frac{\partial w^{G^{*}}}{\partial I}<0, \frac{\partial p^{G^{*}}}{\partial \mu}>0, \frac{\partial p^{G^{*}}}{\partial \alpha}>0, \frac{\partial p^{G^{*}}}{\partial I}<0 \text { 。Prove that. }
$$

Proposition 2 shows that the change of wholesale price and retail price closely related with the consumer preference parameter for low carbon $\mu$, market size $\alpha$ and investment size parameter $I$, i.e. consumers low carbon preference, market size growth of demand will push up the wholesale price and retail price, but with the scale of emission reduction technology investment larger, economies of scale will be produced, that will makes the wholesale price and retail price drop.

\section{B. Profitability comparison}

Proposition 3:when $8 \beta I>\mu^{2}$, there are $\pi_{m}{ }^{G}>\pi_{m}{ }^{N}, \pi_{r}{ }^{G}>\pi_{r}{ }^{N}, \pi^{G}>\pi^{N}$ 。 
Proof: According to （7）、（14）, there is:

$$
\pi_{m}{ }^{G}-\pi_{m}{ }^{N}=\frac{\mu^{2}\left(\alpha-\beta c_{m}-\beta c_{r}\right)^{2}}{8 \beta\left(8 \beta I-\mu^{2}\right)}>0 \quad \pi_{r}{ }^{G}-\pi_{r}{ }^{N}=\frac{\mu^{2}\left(16 \beta I-\mu^{2}\right)\left(\alpha-\beta c_{m}-\beta c_{r}\right)^{2}}{16 \beta\left(8 \beta I-\mu^{2}\right)^{2}}>0,
$$

Prove that.

Proposition 3 shows that when consumers have low carbon product preferences, relative to the not implementation of low carbon technology innovation, manufacturers implementing low carbon technology innovation can make manufacturer and retailer's profits growth. However, apparently this result is by the consumer with the premise of low-carbon product preferences constraints. at the present stage because of China's relevant legislation and consumer awareness is relatively weak, resulting in low carbon practice effect is not ideal in our country enterprise.

Proposition 4:when $8 \beta I>\mu^{2}, \pi_{m}{ }^{G}$ and $\pi_{r}{ }^{G}$ increases with the increasing of $\mu$ or $\alpha$, and decrease with the increasing of $I$.

Proof: According to (7) 、 (14), there is:

$$
\frac{\partial \pi_{m}{ }^{G}}{\partial \mu}>0, \frac{\partial \pi_{m}{ }^{G}}{\partial \alpha}>0, \frac{\partial \pi_{m}{ }^{G}}{\partial I}<0, \frac{\partial \pi_{r}{ }^{G}}{\partial \mu}>0, \frac{\partial \pi_{r}^{G}}{\partial \alpha}>0, \frac{\partial \pi_{r}^{G}}{\partial I}<0 \text {.Prove that. }
$$

Proposition 4 shows that the change of manufacturers and retailers' profits closely related with low carbon preference parameter of consumers $\mu$, market size $\alpha$ and investment size parameter $I$.namely consumers low carbon preference and market size growth of demand will push up manufacturers and retailers' profits, but with the scale of emission reduction technology investment scale larger, economies of scale will be produced,that makes the manufacturers and retailers' profits drop.

\section{Mandatory carbon emissions constraints comparison}

When manufacturers don't implement emission reduction technology innovation, based on the assumption that $E=h-l \theta$,in this case $\theta=0$, then there is $E^{N}=h$, the (6) into manufacturer carbon constraint function $E^{N} D\left(p^{N}\right) \leq E_{\text {cap }}$, can be obtained: $E_{\text {cap }} \geq \frac{h\left(\alpha-\beta c_{m}-\beta c_{r}\right)}{4}$

When manufacturers implement emission reduction technology innovation, based on the assumption $E^{G}=h-l \theta^{G}$,(13) into manufacturer carbon constraint function $E^{G} D\left(p^{G}, \theta^{G}\right) \leq E_{c a p}$, can be obtained $E_{\text {cap }} \geq \frac{2 \beta I\left(\alpha-\beta c_{m}-\beta c_{r}\right)\left(h-l \theta^{G}\right)}{8 \beta I-\mu^{2}}$,Equation (12) into $\theta^{G^{*}}=\frac{\mu\left(\alpha-\beta c_{m}-\beta c_{r}\right)}{8 \beta I-\mu^{2}}$, can be obtained: $E_{a p} \geq \frac{2 \beta I\left(\alpha-\beta c_{m}-\beta c_{r}\right)\left[h\left(8 \beta I-\mu^{2}\right)-l \mu\left(\alpha-\beta c_{m}-\beta c_{r}\right)\right]}{\left(8 \beta I-\mu^{2}\right)^{2}}$

Proposition 5: when meeting the conditions that $8 \beta I>\mu^{2}, \frac{\alpha-\beta c_{m}-\beta c_{r}}{8 \beta I-\mu^{2}} \geq \frac{h \mu}{8 \beta I l}$, can both satisfy the consumer demand, and ensure that the manufacturer implementation of emission reduction technology innovation carbon emissions below the not implementation of carbon emission reduction technology innovation. In this case the policy gives the manufacturer's optimal value range for carbon emissions cap, that is:

$E_{\text {cap }} \in\left[\frac{2 \beta I\left(\alpha-\beta c_{m}-\beta c_{r}\right)\left[h\left(8 \beta I-\mu^{2}\right)-l \mu\left(\alpha-\beta c_{m}-\beta c_{r}\right)\right]}{\left(8 \beta I-\mu^{2}\right)^{2}}, \frac{h\left(\alpha-\beta c_{m}-\beta c_{r}\right)}{4}\right]$

Proof: according to the (15) and (16), if the manufacturer want to ensure that the implementation of emission reduction technology innovation carbon emissions is less than the not implementation of carbon emission reduction technology innovation.

$$
\frac{h\left(\alpha-\beta c_{m}-\beta c_{r}\right)}{4} \geq \frac{2 \beta I\left(\alpha-\beta c_{m}-\beta c_{r}\right)\left[h\left(8 \beta I-\mu^{2}\right)-l \mu\left(\alpha-\beta c_{m}-\beta c_{r}\right)\right]}{\left(8 \beta I-\mu^{2}\right)^{2}} \text {,i.e. } \frac{\alpha-\beta c_{m}-\beta c_{r}}{8 \beta I-\mu^{2}} \geq \frac{h \mu}{8 \beta I l} \text {. At this }
$$

point, in order to make policy to be effective of encouraging technological innovation, the policy gives the manufacturer's optimal value range for carbon emissions cap, that is: 


$$
E_{c a p} \in\left[\frac{2 \beta I\left(\alpha-\beta c_{m}-\beta c_{r}\right)\left[h\left(8 \beta I-\mu^{2}\right)-l \mu\left(\alpha-\beta c_{m}-\beta c_{r}\right)\right]}{\left(8 \beta I-\mu^{2}\right)^{2}}, \frac{h\left(\alpha-\beta c_{m}-\beta c_{r}\right)}{4}\right] \text { Prove that. }
$$

Proposition 5 show that when meet certain conditions, can both satisfy the consumer demand, and ensure the manufacturer implementation of emission reduction technology innovation carbon emissions be less than the not implementation of carbon emission reduction technology innovation. In addition, the policy gives the manufacturer's optimal carbon emissions ceiling exists an limit range, flexible value in this range, can achieve the effect of policies to encourage technological innovation and emission reduction

\section{Examples}

Here there is an automobile engine manufacturer, for example. Assuming that manufacturers unit production cost of $c_{m}=10000$ yuan, retailers' cost of sales is $c_{r}=1000$ yuan. Consumer demand function is for $D(p, \theta)=100000-5 p+3000 \theta, E=100-80 \theta$, manufacturers' reduction technology investment function is $C=10000000 \theta^{2}$.

When manufacturers do not implement emission reduction technology innovation, will be: $w^{N^{*}}=14500$ yuan, $\quad p^{N^{*}}=17750$ yuan, $D\left(p^{N}\right)=11250 \quad$ table, $\pi_{m}{ }^{N}=50625000 \quad$ yuan, $\pi_{r}^{N}=25312500$ yuan, $\pi^{N}=\pi_{m}{ }^{N}+\pi_{r}{ }^{N}=75937500$ yuan. When manufacturers implement emission reduction technology innovation, will be: $w^{G^{*}}=14604$ yuan, $p^{G^{*}}=17905$ yuan, $\theta^{G^{*}}=34.5 \% ; D\left(p^{G}, \theta^{G}\right)=11509$ table, $D\left(p^{G}, \theta^{G}\right)=11509$ yuan, $\pi_{r}^{G}=26491192$ yuan, $\pi^{N}=\pi_{m}{ }^{N}+\pi_{r}^{N}=78281473$ yuan.

According to the comparison of the example data, it is clear that Proposition 1 and Proposition 3 is established.According to proposition 5, the example data $\frac{\alpha-\beta c_{m}-\beta c_{r}}{8 \beta I-\mu^{2}}=\frac{45}{391000}>\frac{h \mu}{8 \beta I l}=\frac{3}{320000}$

When manufacturers do not implement emission reduction technology innovation needed $E_{\text {cap }} \geq 1125000$; When manufacturers implement emission reduction technology innovation needed $E_{\text {cap }} \geq 833000$; the policy gives the manufacturer's optimal carbon emissions ceiling should be in the range $E_{c a p} \in[833000,1125000]$, The specific values should be based on the actual situation.

\section{Conclusion}

In the meet certain conditions, manufacturers to implement emission reduction technology innovation can make the price of manufacturers and retailers and profits higher than not innovation, but can be affected by low carbon preference parameters of consumer $\mu$, market scale $\alpha$ and investment size parameter $I$, the policy to the manufacturer's optimal value range for carbon emissions cap is a threshold value, only in this interval values the policy can make the effection of encouraging technological innovation and emission reduction. This paper considering the mandatory cuts policy constraints, only consider the carbon emissions control constraints, actually carbon tax constraints in practice also is very common. in addition, analysis model can also be extended to the products or manufacturer's competition situation, these can be as the further research direction.

\section{References}

[1] Baris Yalabik, Richard J. Fairchild. Customer, regulatory, and competitive pressure as drivers of environmental innovation[J].International Journal of Production Economics, 2011,2(131):519-527.

[2] Guowei Hua, T.C.E. Cheng, Shouyang Wang. Managing carbon footprints in inventory management [J].International Journal of Production Economics,2011,2(132):178-185.

[3] S. Du, F. Ma, Z. Fu, L. Zhu, J. Zhang. Game-theoretic analysis for an emission-dependent supply chain in a 'cap-and-trade' system[J].Annals of Operations Research,2011(9):1-15.

[4]Debabrata Ghosh, JanatShah. A comparative analysis of greening policies across supply chain structures[J].International Journal of Production Economics,2012,135(4):568-583.

[5] Pim Heijnen, Allard van der Made. A signaling theory of consumer boycotts[J].Journal of Environmental Economics and Management,2012,3(63):404-418. 